\title{
The theoretical interpretation of the Linear/circularly polarized light- induced molecular orientation of the azo group-containing trans-Achiral Schiff base dinuclear complex composite material
}

\author{
Nobumitsu Sunaga1, a , Takashiro Akitsu1 ${ }^{1}$, b, Taro Konomi² and Masahiro Katoh ${ }^{2}$ \\ 1 Tokyo University of Science, Faculty of Science, Department of Chemistry, 162-8601 1-3 Kagurazaka, Shinjuku-ku, Tokyo, Japan \\ 2 Institute for Molecular Science, UVSOR, 444-8585 38 Nishigonaka, Myodaiji, Okazaki, Aichi, Japan \\ a Author to whom correspondence should be addressed; E-Mail: 1317707@ed.tus.ac.jp; Tel.: +81-3-5228-8271. \\ ${ }^{b}$ Author to whom correspondence should be addressed; E-Mail: akitsu@rs.kagu.tus.ac.jp; Tel.: +81-3-5228-8271; Fax: +81-3-5261-4631.
}

\begin{abstract}
We have investigated linearly polarized UV and circularly polarized UV light-induced molecular orientation, namely increasing optical anisotropy of newly synthesized AZ containing chiral Schiff base Ni (II), Cu (II) and $\mathrm{Zn}$ (II) complexes, as organic/inorganic hybrid materials in polymethylmethacrylate (PMMA) cast films. The driving force and reason for polarized UV light-induced molecular orientation of AZ is so-called Weigert effect. The increase of the dichroic was measured with polarized absorption electronic spectra. In addition, we study on newly synthesized composites of AZ containing chiral Schiff base metal complexes with AZ to irradiate with not only linearly but also circularly polarized UV light (Wavelength selective $\left(260,318\left(\pi-\pi^{*}\right.\right.$ of AZ) and $\left.380 \mathrm{~nm}\right)$ and continuous wavelength). In addition, we comparison between simulated by TD-DFT and experimental spectra of electronic and CD spectra, and we calculated dipole moments by TD-DFT. Furthermore, we are using the CD spectrum after circularly polarized light UV irradiation, tried to observation change of supramolecular chiral order of the complexes.
\end{abstract}

\section{Introduction}

In our previous study, by using typical Mn12 singlemolecule magnet ${ }^{1}$ and AZ in PMMA cast films, we could observe switching of AC susceptibility in association with photoisomerization of $\mathrm{AZ}$ at $3 \mathrm{~K} .{ }^{2} \mathrm{C} 1 \mathrm{~s}$ and $\mathrm{O} 1 \mathrm{~s}$ peaks of PMMA could be observed in XPS of the hybrid material, while Mn2p1/2,3/2 peaks, those were shown as bulk Mn12, could not be observed at the surface of PMMA. Which suggests that all components are contained inside the PMMA films (not surface) ${ }^{3}$ Therefore, we employed polarized electronic spectroscopy to measure aggregation and molecular orientation of the components of hybrid materials. We have investigated polarized electronic spectra of hybrid material of Mn12 and AZ in PMMA cast film after alternate UV and visible light irradiation for $3 \min \pi-\pi$ * and $\mathrm{n}-\pi *$ bands suggested increasing of optical anisotropy accompanying with reversible photoisomerizaion of $\mathrm{AZ}$, which may have resulted in previously reported reversible increasing and decreasing of ac magnetic susceptibility. Furthermore, increasing optical anisotropy could be also observed for hybrid materials composed of chiral trans-type Schiff base $\mathrm{Co}(\mathrm{II}), \mathrm{Ni}(\mathrm{II}), \mathrm{Cu}(\mathrm{II})$, and $\mathrm{Zn}(\mathrm{II})$ complexes and $\mathrm{AZ}$ in PMMA cast films after alternate irradiation of polarized UV and visible light ${ }^{4}$ In order to elucidate intermolecular interactions by chiral recognition between components effects of conformation of ligands, we prepared and measured hybrid materials composed of enantiomers or diasteromers of Schiff base Mn(III) complexes and AZ in PMMA cast films. The results suggested that molecular recognition and intermolecular interactions depend on matrix in which $\mathrm{Mn}(\mathrm{III})$ complexes are dispersed or co-existing solutes in the matrix.$^{5}$ Organic/inorganic hybrid materials composed of photochromic dyes such as AZ and inorganic coordination compounds have been studied widely as a key technology for optical (magnetic) data recording. One of the ad-vantages of organic/inorganic hybrid materials may be facility of designing supramolecular multifunctional materials, for example metal complexes incorporating azo-ligands with large dichromic ratios. ${ }^{6-8}$

We also focused on photo-tuning of optical anisotropy of AZ, namely polarized UV light-induced alignment caused by selected photoisomerization and molecular reorientation of AZ (Weigert effect) ${ }^{9-11}$. We measured a CD spectrum each of organic/inorganic hybrid materials and invested photo-induced dichroism. ${ }^{12-14}$ In addition, we discussed the relations between degree of molecular orientation and electric transition dipole moments.

Recently we have investigated linearly polarized UV light induced molecular orientation of chiral Schiff base nickel(II), copper(II) and zinc(II) diunclear complexes in

a,b Corresponding author: 1317707@ed.tus.ac.jp, akitsu@rs.kagu.tus.ac.jp 
organic/inorganic hybrid materials containing azocompounds in a PMMA matrix. Weigert effect makes it parallel orientation of solutes and which was observed optical dichroism with polarized spectroscopy and was discussed with TD-DFT. ${ }^{15-21}$ However, we don't deeply understand the research still organic/inorganic hybrid materials of AZ chiral Schiff base metal complexes for emitting circularly polarized UV light.

Here, we synthesized AZ containing achiral Schiff base $\mathrm{Ni}(\mathrm{II}), \mathrm{Cu}(\mathrm{II})$ and $\mathrm{Zn}$ (II) diunclear complexes (Fig. 1). So, we produced the organic/inorganic hybrid materials by placing the complexes in PMMA film. In addition, we study on newly synthesized composites of achiral Schiff base metal complexes with AZ to irradiate with not only linearly but also circularly polarized UV light (Wave-length selective $\left(280 \mathrm{~nm}, 318 \mathrm{~nm}\left(\pi-\pi^{*}\right.\right.$ of AZ) and $380 \mathrm{~nm}$ ) and continuous wavelength), and examine the di-pole moments and different molecular orientation induced. These complexes effectively contain an azo group to cause the Weigert effect, and hydroxyl group is introduced to enhance the intermolecular interactions PMMA. Furthermore, we attempted to observe conformational changes of metal complexes and supramolecular chiral molecular arrangement for the system by means of CD spectra before and after circularly polarized UV light irradiation.

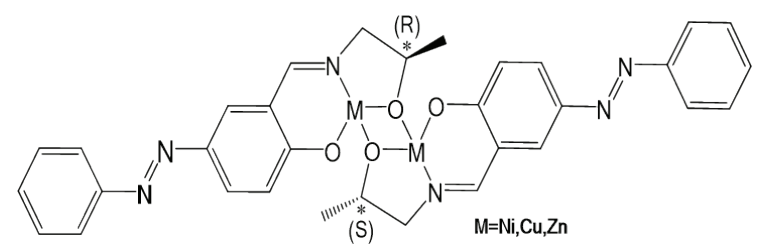

Figure 1. Structures of chiral Schiff base $\mathrm{Ni}(\mathrm{II})(\mathbf{1}), \mathrm{Cu}(\mathrm{II})(2)$, and $\mathrm{Zn}(\mathrm{II})(\mathbf{3})$ diunclear complexes.

\section{Experimental}

\subsection{General procedure}

Chemicals of the highest commercial grade available (solvents from Kanto Chemical, organic compounds from Tokyo Chemical Industry and metal sources from Wako) were used as received with-out further purification.

\subsection{Preparation of the organic/inorganic hybrid materials composes}

We synthesized AZ containing achiral Schiff base $\mathrm{Ni}(\mathrm{II}), \mathrm{Cu}(\mathrm{II})$ and $\mathrm{Zn}(\mathrm{II})$ diunclear complexes to prepare organic/inorganic hybrid materials composes. Then Acetone solution (1 $\mathrm{mL}$ ) of 1-3 and acetone solution (2 $\mathrm{mL})$ of PMMA (10\%) were cast onto a slide glass to give rise to PMMA film of hybrid materials 1-3+PMMA.

\subsubsection{Preparation of (di- $\mu$-oxo-bis[2-[[(2- hydroxypropyl)imino- $\quad \kappa \quad$ N]methyl]-4-2- phenyldiazenylphenolato- $\kappa \mathrm{O}] 2-$ )nickel(II) (1)}

aldehyde (0.5651 g,2.5 mmol) dissolved in methanol (60mL), 1-amino-2-propanol (0.1878 g, $2.5 \mathrm{mmol})$ was added dropwise and stirred at $373 \mathrm{~K}$ for $0.5 \mathrm{~h}$ to give orange solution of ligand. Nickel(II) acetate tetrahydrate (0.6822 g $2.5 \mathrm{mmol}$ ) was added to the resulting solution to give brown solution of the complex. After stirring for $2 \mathrm{~h}$, this crude brown compound was filtered. Yield $0.2068 \mathrm{~g}(12 \%)$. Anal. Calc. for C32H30Ni2N6O2; C, 50.92; H, 4.43; N, 9.73. Found: C, 50.52; H, 4.45; N, $10.36 \%$. IR (KBr (cm-1)): 668, 1117, 1313, 1539, 1603 $(\mathrm{N}=\mathrm{N}), 1652(\mathrm{C}=\mathrm{N}), 2922,3440$.

\subsubsection{Preparation of (di- $\mu$-oxo-bis[2-[[(2- hydroxypropyl)imino- $\kappa \quad$ NJmethyl]-4-2- phenyldiazenylphenolato- $\kappa$ O]2-)copper(II)(2)}

aldehyde (0.5651 g,2.5 mmol) dissolved in methanol (60mL), 1-amino-2-propanol (0.1878 g, $2.5 \mathrm{mmol})$ was added dropwise and stirred at $373 \mathrm{~K}$ for $0.5 \mathrm{~h}$ to give orange solution of ligand. Copper(II) acetate dihydrate (0.4993 g $2.5 \mathrm{mmol}$ ) was added to the resulting solution to give green solution of the complex. After stirring for $2 \mathrm{~h}$, this crude green compound was filtered. Yield 0.1053 g (15\%). Anal. Calc. for $\mathrm{C} 32 \mathrm{H} 30 \mathrm{Cu} 2 \mathrm{~N} 6 \mathrm{O} 2 ; \mathrm{C}, 54.96 ; \mathrm{H}$, 3.83; N, 11.7. Found: C, 55.73; H, 4.38; N, 12.12\%. IR $(\mathrm{KBr}(\mathrm{cm}-1)): 668,1112,1386,1558,1607(\mathrm{~N}=\mathrm{N}), 1635$ $(\mathrm{C}=\mathrm{N}), 2923,3446$.

\subsubsection{Preparation of (di- $\mu$-oxo-bis[2-[[(2- hydroxypropyl)imino- $\kappa \quad$ N]methyl]-4-2- phenyldiazenylphenolato- $\kappa$ O]2-)Zinc(II)(3)}

aldehyde (0.5651 g,2.5 mmol) dissolved in methanol (60mL), 1-amino-2-propanol (0.1878 g, $2.5 \mathrm{mmol})$ was added dropwise and stirred at $373 \mathrm{~K}$ for $0.5 \mathrm{~h}$ to give orange solution of ligand. Zinc(II) acetate dihydrate $(0.54875 \mathrm{~g} 2.5 \mathrm{mmol})$ and sodium hydrogen carbonate ware added to the resulting solution to give orange solution of the complex. After stirring for $2 \mathrm{~h}$, this crude orange compound was filtered. Yield $0.0717 \mathrm{~g}(10 \%)$. Anal. Calc. for C32H30N6O4Zn2; C, 57.41; H, 4.31; N, 12.12. Found: C, 57.45; H, 4.36; N, 12.12\%. IR ( $\mathrm{KBr}$ $(\mathrm{cm}-1)):$ 688, 1114, 1390, 1472, $1593(\mathrm{~N}=\mathrm{N}), 1635$ $(\mathrm{C}=\mathrm{N}), 2965,3445$.

\subsection{Physical measurement}

Elemental analyses were carried out with a PerkinElmer 2400 II CHNS/O analyzer at Tokyo University of Sience.IR spectra were recorded on a JASCO FT-IR 4200 plus spectrophotometer. UV-vis absorption spectra were recorded on a JASCO V-570 or V-570 UV/VIS/NIR spectrophotometer equipped with a polarizer at $298 \mathrm{~K}$. CD spectra were recorded on $\mathrm{s}$ JASCO J-820 spectrophotometer at 298K.Photo-irradiation was carried out with D2 light source for 200-350nm (with a visible cut filter) and Xe light source $350-800 \mathrm{~nm}$ (with a UV cut filter) with a polarizer. Photo-illumination were carried out using a lamp with optical filters (UV $\lambda=200-400$ $\mathrm{nm}$ ) leading to a sample by using optical fibers, quarter 
wave plate (SIGMA KOKI) and polarizer through optical filters.

\subsection{Physical measurement in UVSOR}

Elemental analyses were carried out with a PerkinElmer 2400 II CHNS/O analyzer at Tokyo University of Sience.IR spectra were recorded on a JASCO FT-IR 4200 plus spectrophotometer. UV-vis absorption spectra were recorded on a JASCO V-570 or V-570 UV/VIS/NIR spectrophotometer equipped with a polarizer at $298 \mathrm{~K}$. CD spectra were recorded on S JASCO J-820 spectrophotometer at 298K.Photo-irradiation was carried out with D2 light source for 200-350nm (with a visible cut filter) and Xe light source $350-800 \mathrm{~nm}$ (with a UV cut filter) with a polarizer. Photoillumination were carried out using a lamp with optical filters (UV $\lambda=200-400$ $\mathrm{nm}$ ) leading to a sample by using optical fibers, quarter wave plate (SIGMA KOKI) and polarizer through optical filters.

\subsection{Computational methods}

All calculations were perfumed using the Gaussian 09W software Revision D.10 (Gaussian, Inc.). ${ }^{15}$ The gas phase geometry optimizations were carried out using TDDFT with B3LYP functional. The vertical excitation energy was calculated with the Lanl2dz for $\mathrm{Ni}$ and $\mathrm{Cu}$ and with the $6-31+\mathrm{G}$ (d) basis set for $\mathrm{H}, \mathrm{C}, \mathrm{N}, \mathrm{O}$, and method based on the singlet ground state geometry.

\section{Results and Discussion}

\subsection{Electronic and CD spectra of complexes}

Fig.2. shows comparison between simulated and experimental spectra of electronic and $\mathrm{CD}$ spectra to confirm reliable band assignment. In the case of experimental electronic spectra, $d-d$ bands appear at 689 $\mathrm{nm}$ and $684 \mathrm{~nm}$ for achiral $\mathrm{Ni}(\mathrm{II})$ and $\mathrm{Cu}$ (II) diunclear complexes respectively. Also, $\pi-\pi *$ bands appear at $285 \mathrm{~nm}, 282 \mathrm{~nm}$ and $286 \mathrm{~nm}$ and $\mathrm{n}-\pi^{*}$ bands appear at $369 \mathrm{~nm}, 342 \mathrm{~nm}$ and $352 \mathrm{~nm}$ for achiral Ni(II), Cu(II) and $\mathrm{Zn}$ (II) diunclear complexes, respectively. In the case of simulated spectra, d-d bands appear at $692 \mathrm{~nm}$ and 701 $\mathrm{nm}$ and transition electric dipole moment is evaluated to be $(-0.0836,-0.3985,-0.6970)$ and $(-1.0799,0.3828$, $0.0029)$ with oscillator strength of $0.8072 \mathrm{D}$ and 1.1458 $\mathrm{D}$ for $\mathrm{Ni}(\mathrm{II})$ and $\mathrm{Cu}(\mathrm{II})$ complexes, respectively. Also, $\pi$ - $\pi$ * bands appear at $385 \mathrm{~nm}, 392 \mathrm{~nm}$ and $381 \mathrm{~nm}$ and n$\pi *$ bands appear at $469 \mathrm{~nm}, 442 \mathrm{~nm}$ and $453 \mathrm{~nm}$ for achiral $\mathrm{Ni}(\mathrm{II}), \mathrm{Cu}(\mathrm{II})$ and $\mathrm{Zn}(\mathrm{II})$ diunclear complexes, respectively. Moreover, $\mathrm{n}-\pi^{*}$ and $\pi-\pi *$ bands appear at $486 \mathrm{~nm}$ and $329 \mathrm{~nm}$ and transition electric dipole moment is evaluated to be $(0,0,0)$ and $(-0.1230,-2.8902$, 0 ) with oscillator strength of 0.0000 and 0.7726 for the trans-form of AZ. The corresponding $n-\pi *$ and $\pi-\pi^{*}$ bands appear at $482 \mathrm{~nm}$ and $301 \mathrm{~nm}$ and transition electric dipole moment is evaluated to be $(-0.7441,0$, $0.2159)$ and $(0.7979,0,0.2324)$ with oscillator strength of 0.0378 and 0.0696 for the cis-form of AZ. ${ }^{24}$ According to preceding studies on the related compounds, the optimized structures and assignments of transitions for $\mathbf{1}$, $\mathbf{2}$ and $\mathbf{3}$ are reasonable.

Selected predominant peaks are assigned to transitions between molecular orbitals: for $1,662 \mathrm{~nm}(\mathrm{~d}-\mathrm{d}$ band)(HOMO (166) to LUMO+2 (169)), $385 \mathrm{~nm}(\pi-\pi$ * band)(HOMO-1 (154) to LUMO+1 (168)), and $469 \mathrm{~nm}$ (n- $\pi$ * band)(HOMO-11 (150) to LUMO+5 (172)); for $\mathbf{2}$, $701 \mathrm{~nm}$ (d-d band)(HOMO-14 (153) to LUMO (168)), $392 \mathrm{~nm}$ ( $\pi-\pi *$ band)(HOMO-8 (159) to LUMO (162)), and $442 \mathrm{~nm}$ (n- $\pi *$ band)(HOMO (167) to LUMO+5 (172)); for 3, $299 \mathrm{~nm}$ ( $\pi-\pi^{*}$ band)(HOMO-5 (155) to LUMO (161)), $381 \mathrm{~nm}$ (n- $\pi *$ band) (HOMO (160) to LUMO+1 (162)).
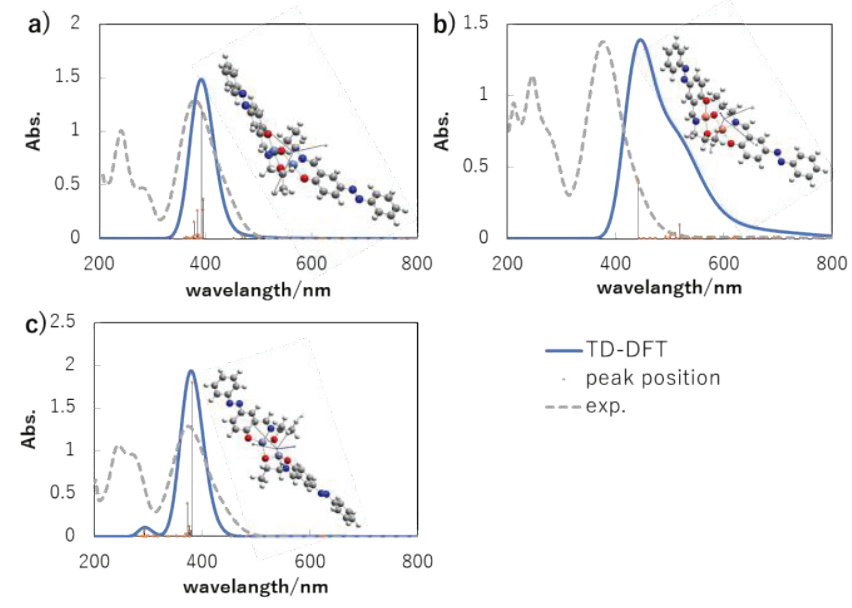

Figure 2. Simulated (blue(TD-DFT)) and experimental (grey) electronic spectra of $\mathbf{1}$ (a), 2 (b) and $\mathbf{3}$ (c) with optimized structures.

\subsection{Polarized absorption spectra of azo- containing hybrid materials after linearly polarized UV light irradiation}

Fig. 3 shows polarized absorption spectra and circular diagrams of angular dependence of absorbance of $\pi-\pi^{*}$ band at $280 \mathrm{~nm}$ and $\mathrm{n}-\pi *$ band at $380 \mathrm{~nm}$ of AZ group and d-d band at $689 \mathrm{~nm}$ of 1 for 1+PMMA. The decrease in absorbance of the $\pi-\pi^{*}$ and $n-\pi *$ at initial is due to photoisomerization. Optical anisotropy increased as time proceed. The circular diagrams suggest that reorientation of AZ effects on relatively rich optical anisotropy observed by $\pi-\pi *$ and $n-\pi *$ bands. Polarized UV light irradiation induced optical anisotropy with de-creasing intensity of $\pi-\pi^{*}$ band significantly. Fig. 4 shows polarized absorption spectra and circular diagrams of angular dependence of absorbance of $\pi-\pi^{*}$ band at 280 $\mathrm{nm}$ and $\mathrm{n}-\pi^{*}$ band at $380 \mathrm{~nm}$ of AZ and d-d band at 684 nm of 2 for 2+PMMA. Initial change of $\pi-\pi^{*}$ band feature is also observed because of AZ occurs cis-trans photoisomerization. Fig. 5 shows polarized absorption spectra and circular diagrams of angular dependence of 
absorbance of $\pi-\pi *$ band at $280 \mathrm{~nm}$ and $\mathrm{n}-\pi *$ band at $380 \mathrm{~nm}$ of AZ. The order of enhancing dichroism is 2+PMMA $>$ 3+PMMA $>$ 1+AZ+PMMA.

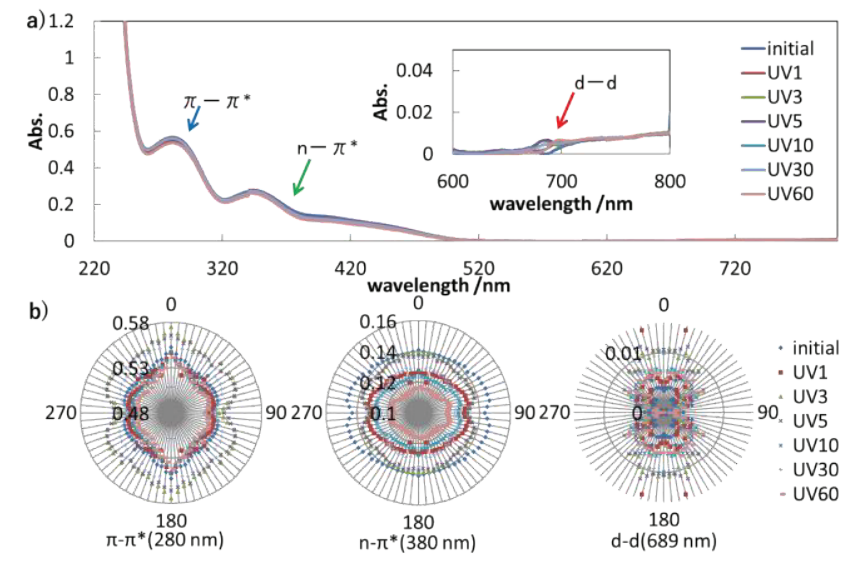

Figure 3. (a) Polarized absorption spectra of 1+PMMA with 0 degree showing predominant peaks before and after UV light irradiation for $60 \mathrm{~min}$. (b) Circular diagram of angular dependence of absorbance of the predominant peaks polarized absorption spectra of 1+PMMA after UV light irradiation for 0 ,

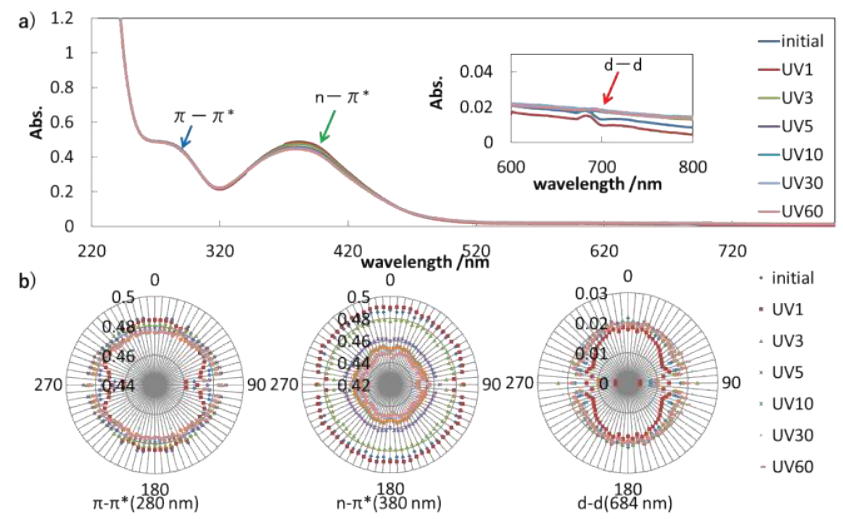

$1,3,5,10,30$ and 60 min respectively ads. bands.

Figure 4. (a) Polarized absorption spectra of 2+PMMA with 0 degree showing predominant peaks before and after UV light irradiation for $60 \mathrm{~min}$. (b) Circular diagram of angular dependence of absorbance of the predominant peaks polarized absorption spectra of 2+PMMA after UV light irradiation for 0 , $1,3,5,10,30$ and 60 min respectively ads. bands.

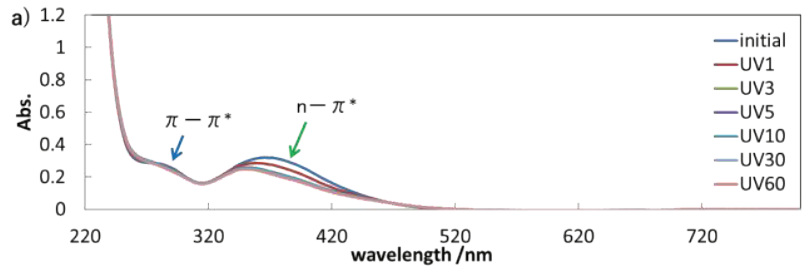

b)
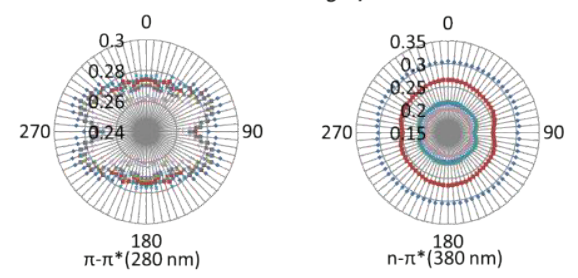

- initial

- UV1

UV3

UV 5

UV30

UV60

Figure 5. (a) Polarized absorption spectra of $3+$ PMMA with 0 degree showing predominant peaks before and after UV light irradiation for $60 \mathrm{~min}$. (b) Circular diagram of angular dependence of absorbance of the predominant peaks polarized absorption spectra of 3+PMMA after UV light irradiation for 0 , $1,3,5,10,30$ and 60 min respectively ads. bands.

\subsection{Parameters for optical anisotropy}

The degree of photoinduced optical anisotropy (spectral dichroism) of absorption electronic spectra can be described commonly by these two parameters:

$$
\begin{aligned}
& \quad S=(\text { Aparrallel }- \text { Aperpendicular }) \\
& \text { /(2Aperpendicular }+ \text { Aparrallel }) \\
& R=\text { Aperpendicular / Aparrallel }
\end{aligned}
$$

Where Aperpendicular and Aparrallel values denote absorbance measured with the measuring polarizers perpendicular or parallel to electric vector of irradiation polarized light. Ideal isotropic systems of $\mathrm{S}=0$ and $\mathrm{R}=1$ and both $\mathrm{S}$ and $\mathrm{R}$ parameters are changed as increasing dichroism by molecular alignment. General treatment has been explained in our previous articles for cis-trans isomerizaiton as well as Weigert effect. The $\mathrm{S}$ values are summarized in Tables respectively. Hereafter we discuss them in a qualitative level.

Table 1. The $\mathrm{S}$ values of anisotropic parameters polarized absorption spectra for $\mathbf{1 , 2}$ or $\mathbf{3}+$ PMMA after linearly polarized UV light irradiation.

\begin{tabular}{cccccccc}
\hline \multicolumn{2}{c}{ UV irradiation (min.) } & initial & $\mathbf{1}$ & $\mathbf{3}$ & $\mathbf{5}$ & $\mathbf{1 0}$ & $\mathbf{3 0}$ \\
\hline 1+PMMA & $280 \mathrm{~nm}, \pi-\pi^{*}(\mathbf{A Z})$ & 0 & -0.00069 & 0.00886 & 0.000206 & 0.002015 & 0.005864 \\
& $380 \mathrm{~nm}, \mathrm{n}-\pi^{*}(\mathbf{A Z})$ & 0 & -0.00277 & 0.023518 & 0.01895 & 0.009389 & 0.034243 \\
& $689 \mathrm{~nm}, \mathrm{n}-\pi^{*}(\mathbf{1})$ & 0 & -0.09636 & -0.52621 & -0.55414 & -0.49733 & -0.16811 \\
$2+$ PMMA & $280 \mathrm{~nm}, \pi-\pi^{*}(\mathbf{A Z})$ & 0 & 0.00535 & -0.00899 & -0.01197 & -0.01036 & -0.01004 \\
& $380 \mathrm{~nm}, \mathrm{n}-\pi^{*}(\mathbf{A Z})$ & 0 & 0.002101 & -0.0051 & -0.00453 & -0.00546 & -0.00589 \\
& $684 \mathrm{~nm}, \mathrm{n}-\pi^{*}(\mathbf{2})$ & 0 & 0.019426 & -0.10287 & -0.04259 & -0.02426 & -0.04989 \\
$3+$ PMMA & $280 \mathrm{~nm}, \pi-\pi^{*}(\mathbf{A Z})$ & 0 & 0.008322 & 0.009576 & 0.00836 & 0.013522 & 0.00445 \\
& $380 \mathrm{~nm}, \mathrm{n}-\pi^{*}(\mathbf{A Z})$ & 0 & 0.018025 & 0.008556 & 0.012543 & 0.010613 & 0.009471 \\
& No d-d band & & & & & & \\
& & & & & & &
\end{tabular}

\subsection{Discuss about linear polarized light irradiation}

For the change of the absorption spectrum of after linearly polarized UV light irradiation, knowledge regarding molecular orientation will be obtained conformation, from $\mathrm{S}$ value of $\pi-\pi^{*}$ band, a change in the coordination structure from $\mathrm{d}-\mathrm{d}$ band. For the azocontaining systems $(\mathbf{1}, \mathbf{2}$, or $\mathbf{3}+\mathrm{PMMA})$, azo-containing for complexes can be influenced by Weigert effect of AZ. Metal ions providing rigid coordination environment are effective to increase molecular orientation caused by Weigert effect. ${ }^{22-24}$ According to Table 1, we can derive the following information associated with molecular orientations with intermolecular interactions in these organic/inorganic hybrid materials. The order of the effect, $2+$ PMMA $>\mathbf{3}+$ PMMA $>\mathbf{1}+$ PMMA for $\pi-\pi *$ bands and $n-\pi *$ bands, $2+$ PMMA $>1+$ PMMA for $d-d$ bands Result in the smallest degree of increasing optical anisotropy in both of $n-\pi *$ and $\pi-\pi *$ accompanying with Weigert effect of AZ. Reducing direct transmission of molecular alignment from AZ by rigidity of metal complexes due to $\mathrm{Zn}$ (II) ions is suggested. ${ }^{23,} 25-30$ The degree of increasing optical anisotropy accompanying with Weigert effect of $\mathrm{AZ}$ depends on not shapes of 
chiral ligands but central $\mathrm{Ni}(\mathrm{II}), \mathrm{Cu}(\mathrm{II})$ or $\mathrm{Zn}$ (II) metal ions.

\subsection{Discussion about CD spectra after circularly polarized UV light irradiation}

Figure 6-8 show the CD spectra of 1, 2, or 3+PMMA, after wavelength selective circularly polarized UV light irradiation 5min. Figure 6. shows the CD spectra of 1+PMMA around $380 \mathrm{~nm}$ and $280 \mathrm{~nm}$ exhibited changes before and after wavelength selective circularly polarized UV light irradiation $(280 \mathrm{~nm}, 318 \mathrm{~nm}$ and 380nm) for 1+PMMA. In the $280 \mathrm{~nm}$ UV irradiation, was induced $\mathrm{CD}$ is the largest in two steps of $3,8 \mathrm{~min}$. The $318 \mathrm{~nm}$ UV irradiation, significant induced $\mathrm{CD}$ could not be confirmed. In the $380 \mathrm{~nm}$ UV irradiation, induced CD became the largest in $8 \mathrm{~min}$. In Figure 7 . shows the CD spectra of 2+PMMA, after the $280 \mathrm{~nm}$ UV irradiation, induced $\mathrm{CD}$ also increased in proportion to the irradiation time. In the $318 \mathrm{~nm}$ UV irradiation, induced CD be-came the largest in the irradiated for $3 \mathrm{~min}$. In the $380 \mathrm{~nm} \mathrm{UV}$ irradiation, was induced CD is the largest in two steps of 3, $8 \mathrm{~min}$. Figure 8 . shows the CD spectra of $3+\mathrm{PMMA}$ around $380 \mathrm{~nm}$ and $280 \mathrm{~nm}$ exhibited changes before and after wavelength selective circularly polarized UV light irradiation (260 nm, $318 \mathrm{~nm}$ and 380nm) for 3+PMMA. In the $280 \mathrm{~nm}$ UV irradiation, induced CD became the largest in two stages for $8 \mathrm{~min}$. In the $318 \mathrm{~nm} \mathrm{UV}$ irradiation, significant induced $\mathrm{CD}$ could not be confirmed. In the $380 \mathrm{~nm}$ UV irradiation, it was confirmed that similar induced CD in any irradiation time.
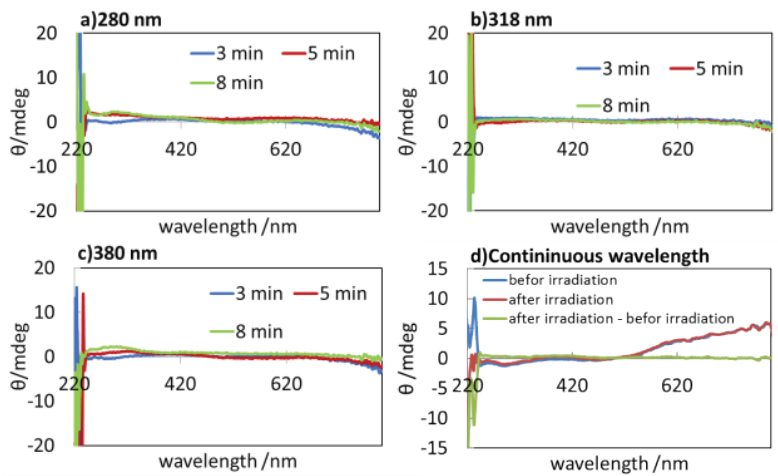

Figure 6. CD spectra of $\mathbf{1}$ after circularly polarized UV light irradiation with respectively $260,318,380 \mathrm{~nm}$ and continuous.
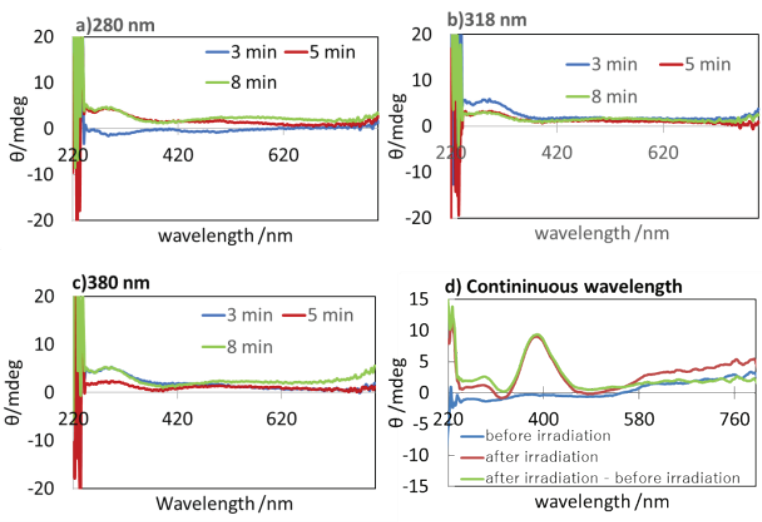

Figure 7. CD spectra of 2 after circularly polarized UV light irradiation with respectively $260,318,380 \mathrm{~nm}$ and continuous.
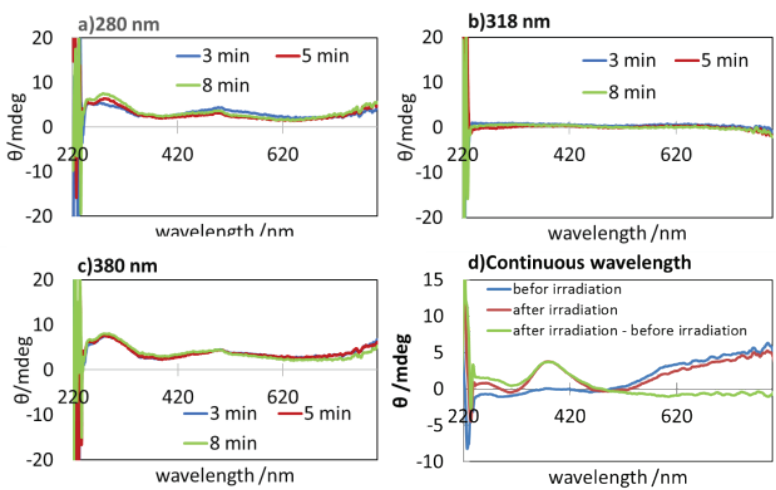

Figure 8. CD spectra of 3 after circularly polarized UV light irradiation with respectively $260,318,380 \mathrm{~nm}$ and continuous.

Continuous wavelength circularly polarized UV light irradiation, the difference between the CD spectra be-fore and after irradiation of circular polarization, is that peak was observed at around $400 \mathrm{~nm}$. This peak was different from peak of the CD spectra due to the chiral complex molecule attributed in TD-DFT calculation. It is considered from this fact, that peak emerging from it is the peak of supramolecular chiral (helical) order and is not derived from the complex itself. There was change in the peak position in the chiral and achiral, and chiral strength had become stronger. On the other hand, using a light source having a narrow wavelength width in UVSOR (Wavelength selective $(280,318(\pi-\pi *$ of AZ) and $380 \mathrm{~nm}$ ), induced chiral order is caused absorption wavelength selective. Changes in the observed shape of the CD spectra, the induction of supramolecular chirality or conformational change of the molecule can be considered as the cause. Changes in the CD spectra of this time, appeared only change in absorption intensity with increasing the irradiation time, which suggested that molecules of supramolecular chirality induction by Weigert effect of AZ by circularly polarized light irradiation, rather than the changes in molecular shape. In addition, induced chiral order is caused absorption wavelength selective, which suggests that it is a new knowledge of the radiation source only. Peak shift of appearing by changing the wavelength selective is seen. There was change in the peak position in the chiral and achiral, and chiral strength had become stronger. This is suggested that it is something that those which have a chiral place is easily oriented supramolecular chiral (helical) order is well. The order of this effect is 2+PMMA $>3+$ PMMA $>\mathbf{1}+$ PMMA, and these results suggests that optical anisotropy depends not only on flexibility of coordination environment but also coordination geometries.

\subsection{Discussion about Theoretical interpretation of the optical rotation strength using coupled oscillator model}

For interpretation of supramolecular chirality, we attempt to discuss spatial relationship between AZ molecules assuming dipole-dipole interaction. For this reason, we will apply coupled oscillator model hereaf- 
ter. $^{31-40}$ If only the Coulomb interaction of the chromospheres A between B two can be considered, we assume system dipole interactions between paired the two ones. Now, the Hamiltonian of the system is represented by the equation (3).

$$
\boldsymbol{H}=\boldsymbol{H}_{A}\left(\boldsymbol{r}_{A}\right)+\boldsymbol{H}_{B}\left(\boldsymbol{r}_{B}\right)+\boldsymbol{V}_{A B}
$$

Where Hamiltonian $H A(r A)$ or $H B(r B)$ is of the chromophores $\mathrm{A}$ or $\mathrm{B}, V A B$ is Coulomb interaction potential between the chromophore $\mathrm{A}$ and $\mathrm{B}$. By the wave function of the ground states and excited states of the chromophores $\mathrm{A}$ or $\mathrm{B}$, to introduce electric transition dipole moment, optical rotation strength of the formula is derived as the equation (4).

$$
R_{0 a}=-\left(\frac{2 \pi v_{a} v_{b}}{h c\left(v_{a}^{2}-v_{b}^{2}\right)}\right)\left(\mu_{0 a} \cdot T \cdot \mu_{0 b}\right)\left(R_{A B} \times \mu_{0 a} \cdot \mu_{0 b}\right)
$$

Where $\mathrm{Va}$ or $\mathrm{Vb}$ are absorption frequency, $\mu \mathrm{0a}$ or $\mu$ $0 \mathrm{~b}$ is electric transition dipole moment, $\mathrm{RAB}$ is a distance of $\mathrm{A}$ and $\mathrm{B}, \mathrm{T}$ is parameters. To simplify this, con-sider that both chromospheres are identical, and hereafter assume connecting both two, is orthogonal (Figure 9).

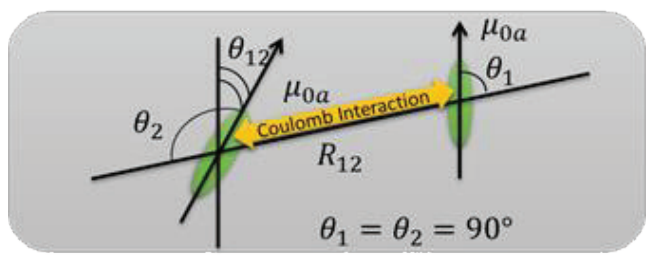

Figure 9. Coulomb interaction between the same chromospheres

Now, from equation (3), the optical rotation strength becomes the following equation (4). Furthermore, we considered as follows. The intensity of $\mathrm{CD}$ bands can be represented by optical rotation strength $\mathrm{R}$. From the $\mathrm{CD}$ spectra measured, $\mathrm{R}$ is deter-mined according to the following equation (6).

$$
\begin{aligned}
& R_{ \pm}=\mp\left(\pi v_{0} / 2 c\right) R_{12} \mu_{0 a}{ }^{2} \sin \theta_{12} \\
& R=2.296 \times 10^{-39} \int_{0}^{\infty}\{\Delta \varepsilon(\sigma) / \sigma\} d \sigma \\
& \approx\left(2.296 \times 10^{-39} / \sigma_{0}\right) \int_{0}^{\infty} \Delta \varepsilon(\sigma) d \sigma \text { (c.g.s) }
\end{aligned}
$$

Where $\sigma$ is the wave number and is the $\sigma 0$ wavenumber of the peaks (the maximum) of the CD spectra. As relational expressions, we calculated from equation (6) optical rotation strength of the induced CD spectra observed. Substituting equation (5), the degree of inclination $(\theta)$ of the dye molecules of interest was evaluated.

Table 4. The angle of optical rotation and strength to transition electric dipole moment of each complex in the $\pi-\pi *$ or $\mathrm{n}-\pi^{*}$ transition states. For simplicity, R12 was assumed to be $0.5 \mathrm{~nm}$ all.

\begin{tabular}{cccc}
\hline & 1+PMMA & 2+PMMA & 3+PMMA \\
\hline$R\left(\mathrm{n}-\pi^{*}\right) / \times 10^{-42}$ c.g.s & 1.64 & 2.26 & 5.7116 \\
$\theta / \times 10^{-40} \mathrm{deg}$ & 38.8 & 60.7 & 3.1234 \\
$\mu 0_{a}\left(n-\pi^{*}\right) /$ Debye & 3.49503972 & 3.657174 & 22.55621 \\
$R\left(\pi-\pi^{*}\right) / \times 10^{-42}$ c.g.s & 1.58 & 1.85 & 1.57291 \\
$\theta / \times 10^{-40}$ deg & 121 & 174 & 215.239 \\
$\mu 0_{a}\left(\pi-\pi^{*}\right) /$ Debye & 1.89947416 & 1.562521 & 1.417491 \\
$R\left(\pi-\pi^{*}\right) / \times 10^{-42}$ c.g.s & 2.76 & 10.5 & - \\
$\theta / \times 10^{-40}$ deg & 457 & 15200 & - \\
$\mu 0_{a}\left(\pi-\pi^{*}\right) /$ Debye & 0.0054 & 0.188345 & - \\
\hline
\end{tabular}

The calculated results indicated that transition dipoles of AZ resulting in large electric dipole transition of $\mathrm{n}-\pi^{*}$ and $\pi-\pi *$ transitions are significantly inclined each other, which was attributed to mutual molecular orientation induced by circularly polarized UV light irradiation. In addition, comparison all 1, 2, or $3+$ PMMA suggested chiral molecular orientation derived from AZ group in complex. Respect to the circularly polarized light irradiation, 2+PMMA was considered a large lightresponsive overall. Further, from the comparison of $n-\pi$ * transition corresponding to $380 \mathrm{~nm}$ circularly polarized light irradiation, 2+PMMA is suggested to have a relatively large photo responsive. From the comparison of theoretical optical rotation strength, optical response of the magnitude Respect to circularly polarized light ultraviolet light was found to be $2+\mathrm{PMMA}>\mathbf{3}+\mathrm{PMMA}>$ 1+PMMA.

\section{Conclusion}

In summary, we have prepared organic/inorganic hybrid materials of azo-containing achiral Schiff base $\mathrm{Ni}(\mathrm{II}), \mathrm{Cu}(\mathrm{II})$, and $\mathrm{Zn}(\mathrm{II})$ complexes in PMMA. Linearly polarized UV light irradiation resulted in increasing optical anisotropy, which was observed with polarized UV-vis spectra (overlapped bands) of $\pi-\pi *$, n- $\pi *$ and $\mathrm{d}-\mathrm{d}$ bands. We discussed the assignment and the origin of $\mathrm{CD}$ bands of hybrid materials of chiral metal complexes and $\mathrm{AZ}$ after irradiation of polarized light by means of TD-DFT calculations. In the PMMA film, the order of this effect is $\mathbf{2}+\mathrm{PMMA}>\mathbf{3}+\mathrm{PMMA}>\mathbf{1}+\mathrm{PMMA}$. The results suggested that differences in optical anisotropy is ascribed to intermolecular interaction with AZ groups due to flexibility of coordination environment of metal centres of the complexes. Circularly polarized UV light irradiation resulted in increasing intensity of CD bands around $380 \mathrm{~nm}$, which suggested induced chiral molecular orientation. Continuous wavelength circularly polarized UV light irradiation resulted in increasing intensity of CD bands, which suggested induced chiral molecular orientation according to calculated dipole moment. On the other hand, using a light source having a narrow wavelength width in UVSOR (Wavelength selective $(280,318(\pi-\pi *$ of $A Z)$ and $380 \mathrm{~nm})$, induced chiral order is caused absorption wavelength selective, which suggests that it is a new knowledge of the radiated 
light. This effect is $\mathbf{2}+\mathrm{PMMA}>\mathbf{3}+\mathrm{PMMA}>\mathbf{1}+\mathrm{PMMA}$, and these results are suggesting that optical anisotropy depends not only on flexibility of coordination environment but also stable coordination geometries. Linearly polarized UV light irradiation resulted in increasing optical anisotropy. It is attributed to the arrangement (not magnitude) of transition electric dipole moments. $\mathrm{Ni}(\mathrm{II})$ is prefer a planar structure and $\mathrm{Cu}(\mathrm{II})$ is taking a flexible structure, but $\mathrm{Zn}(\mathrm{II})$ is prefer a tetrahedral structure for the rigid, which suggests that it is less susceptible to orientation by AZ. We discussed the assignment and the origin of $\mathrm{CD}$ bands of hybrid materials of chiral metal complexes and AZ after polarized light irradiation by TD-DFT calculations. As a result, Respect to the circularly polarized light irradiation, 2+PMMA was considered a large light-responsive overall. Further, from the comparison of $n-\pi *$ transition corresponding to $380 \mathrm{~nm}$ circularly polarized light irradiation, 2+PMMA is suggested to have a relatively large photo responsive. From the comparison of theoretical optical rotation strength, optical response of the magnitude Respect to circularly polarized light ultraviolet light was found to be $2+$ PMMA $>3+$ PMMA $>$ 1+PMMA.

\section{Acknowledgment}

This work was supported by Research Foundation for Opto-Science and Technology. The authors thank Institute for Molecular Science for the use of UVSOR facility (Prof. Hiromasa. Kato and Dr. Taro. Konomi) and CD spectrometer (Mr. Seiji Makita). The Computations were performed using Research Centre for Computational Science, Okazaki, Japan.

\section{References}

1. O. A. Blackburn, B. J. Coe, J. Fielden, M. Helliwell, J. J. W. McDounall and M. G. Hutchings, Inorg. Chem., 49, 9136(2010).

2. A. A. Khandar and Z. Revani, Polyhedron, 18, 129(1998).

3. S. Leclair, L. Mathew, M. Giguere, S. Motallebi, and Y. Zhao, Macromolecules, 36, 9024(2003).

4. Y. Einaga, M. Taguchi, G. Li, T. Akitsu, T. Sugai and O. Sato, Chem. Mater., 15, 8(2003).

5. R. Mikami, M. Taguchi, K. Yamada, K. Suzuki, O. Sato and Y. Einaga, Angew. Chem. Int. Ed., 43, 6135(2004).

6. T. Yamamoto, Y. Umemura, O. Sato and Y. Einaga, Chem. Mater., 16, 1195(2004).

7. Y. Einaga, R. Mikami, T. Akitsu and G. Li, Thin Solid Films, 493, 230(2005).

8. T. Akitsu and A. Yoshida, Curr. Phys. Chem., 1, 76(2011).

9. T. Akitsu and Y. Miura, J. Chem. Chem Eng., 5, 443(2011).

10. Y. Aritake, T. Takanashi, A. Yamazaki and T. Akitsu, Polyhedron, 30, 886(2011).

11. T. Akitsu and T. Itoh, Polyhedron, 2007, 29, 477(2010).
12. Y. Aritake, T. Takanashi, A. Yamazaki and T. Akitsu, Polyhedron, 30, 886(2011).

13. Y. Aritake, T Akitsu, Polyhedron, 31, 278(2012).

14. A. Yamazaki, T. Akitsu, RSC Advances, 2, 2975(2012).

15. M. J. Frisch, G. W. Trucks, H. B. Schlegel, and D. J. Fox, et al., Gaussian, Inc., Wallingford CT, 2009.

16. S. Yamada, Coord. Chem. Rev., 537, 190(1999).

17. A. A. Khandar and Z. Revani, Polyhedron, 18, 129(1998).

18. T. Akitsu, Y. Einaga, Acta Cryst., C60, m640(2004).

19. T. Akitsu, Y. Einaga, Polyhedron, 25, 1089(2006).

20. C. Evans, D. Luneau, J. Chem. Soc., Dalton Trans., 83(2002).

21. H. Sakiyama, H. Okawa, N. Matsumoto and S. Kida, J. Chem. Soc., Dalton Trans., 2935(1990).

22. H. Sakiyama, H. Okawa, N. Matsumoto and S. Kida, Bull. Chem. Soc. Jpn., 64, 2644(1991).

23. M. Ulusoy, H. Karabiyik, R. Kilincarslan, M. Aygun, B. Cetinkaya and S. Garcia-Granda, Struct. Chem., 19, 749(2008).

24. R. S. Forgan, J. E. Davidson, S. G. Galbraith, D. K. Henderson, S. Parsons, P. A. Tasker and F. J. White, Chem.commun., 4049(2008).

25. T. Akitsu and Y. Einaga, Acta. crystallogr., E60, m1555(2004).

26. T. Akitsu and Y. Einaga, Acta. crystallogr., E60, m640(2004).

27. S. Banerjee, A. K. Mukherjee, I. Banerjee, R. L. De Neumann, and L. Louer, Cryst.res.technol., 3, 4815(2005).

28. P. C. Chia, D. P. Freyberg, G. M. Mockler, and E. Sinn, Inorg.chem., 16, 254(1977).

29. J. M. Fernandez-G, C. Ausbun-Valdes and R. A. Toscano Gonzalez-Guerrero, Z.anorg.allg.chem., 633, 1251(2007).

30. D. Hall, S. V. Sheat and TN T. Waters, J. chem.soc.A, 460(1968).

31. T. Akitsu and Y. Einaga, Acta. crystallogr., E60, m1605(2004).

32. T. Akitsu and C. Kominato, InTech, 515(2013).

33. L. Nikolova, T. Todorov, M. Ivanov, F. Andruzzi, S. Hvilsted and P. S. Ramanujam, Opt. Mater., 8, 255(1997).

34. G. Iftime, F. L. Labarthet, A. Natansohn, and Paul Rochon, J. Am. Chem. Soc., 122, 12646(2000).

35. A. Natansohn and P. Rochon, AdV. Mater., 11, 1387(1999).

36. S. Banerjee, A. K. Mukherjee, I. Banerjee, R. L. De Neumann, and L. Louer, Cryst.res.technol., 3, 4815(2005).

37. D. Hall, S. V. Sheat and TN T. Waters, J. chem.soc.A, 460(1968).

38. S. Matile, N. Berova, K. Nakanishi, J. Fleischhauer,R. W. Woody, J. Am. Chem. Soc., 118, 5198 (1996).

39. D. J. Caldwell, H. Eyring, "Theory of Optical Activity", Wiley-Interscience, New York (1971).

40. N.Sunaga, S.Furuya, T.Akitsu, et al., Computational Chemistry: Theory, Methods and Applications, Nova Science Publishers, Inc., 85 (2014). 